\title{
Protocol for Storm Surge Water Level Observations using the Local Environmental Observer Phone Application for Alaska
}

Jacquelyn R. Overbeck
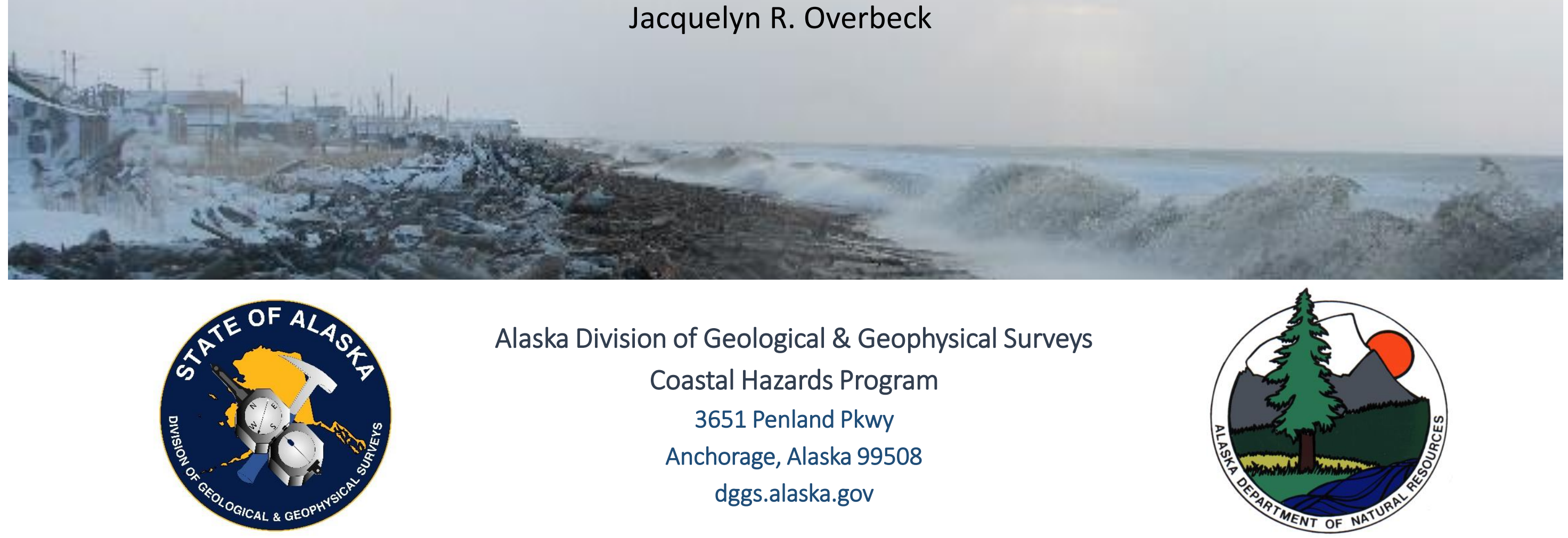

Alaska Division of Geological \& Geophysical Surveys

Coastal Hazards Program

3651 Penland Pkwy

Anchorage, Alaska 99508

dggs.alaska.gov

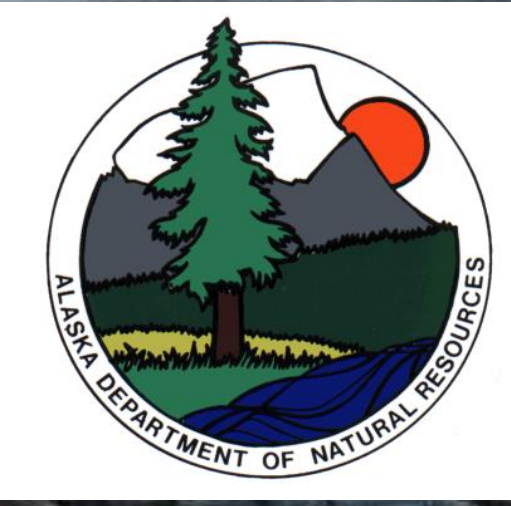




\section{Introduction \& Purpose}

Storm surge is the major cause of flooding and erosion along the western coast of Alaska. Coastal communities that may have been protected by sea ice and landfast ice during past storms are experiencing open oceans during storm seasons, leaving the coastline vulnerable to floodwaters and wave energy.

Although it is commonly recognized that these hazards affect many coastal communities, site-specific data about the characteristics and magnitudes of storm surge remains poor or nonexistent. Gaps in water-level monitoring networks are large, pre-storm instrumentation protocols are not well developed, and post-storm evaluations are difficult and expensive. The paucity of oceanographic instrumentation in Alaska results in minimal measures for coastal scientists and modelers to update storm forecast models and little data to validate the models that already exist. Storm events also often cause damages to infrastructure, which may qualify a community for State or Federal resources to help repair or mitigate future risks (USACE, 2012). However, to qualify for external resources, physical evidence of the damages must be documented. Because of these circumstances, crowd-sourced information has become a necessary and viable option for post-storm impact assessments. This document has been created to inform the public of protocols that can be taken to ensure data quality and usability for the assessments of flood impact after a storm. 


\section{Local Environmental Observer (LEO) Network}

"LEO is a network of local observers and topic experts who share knowledge about unusual animal, environment, and weather events. With LEO, you can connect with others in your community, share observations, raise awareness, and find answers about significant environmental events. You can also engage with topic experts in many different organizations and become a part of a broader observer community." (LEO, 2016)

When an observation is posted on the LEO network, any participant of the LEO network can view that observation. The observation can also be organized by Project type, so that observations about similar processes or events are grouped together. This information is then passed on to scientists or agency officials that serve as the Project Lead. If the observation is entered in a specific way, this information can even be used to extract data from the observation.

For the purpose of measuring the maximum elevations of flooding from storms impacting Alaska, the following information will help an observer to collect an observation in a format that can be converted into data.

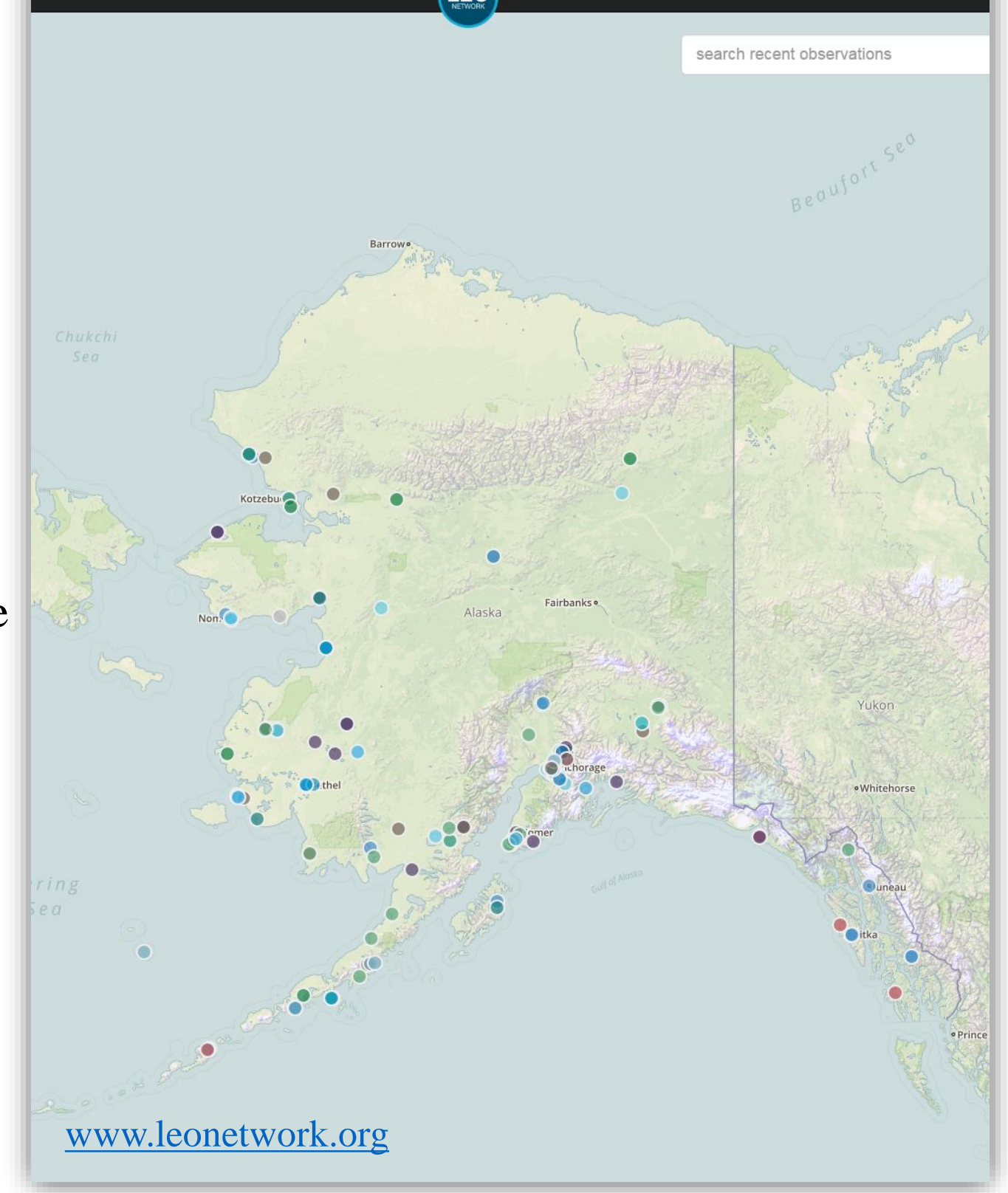




\section{Smartphone Global Positioning System (GPS)}

\section{Accuracy}

Innovations in GPS technology have allowed the majority of people in the U.S. to have the capability to collect and use GPS on a daily basis using smartphones. Smartphones also allow for physical characteristics to be connected automatically to GPS coordinates. Although smartphones are not as accurate as Differential Global Positioning Systems (DGPS; see right), they are readily available and easy to use. In situations where travel to a location may be prohibited by cost or accessibility (such as during a storm event), smartphones are an acceptable option for collecting spatial information. To reduce the spatial uncertainty in coordinate location, certain protocols must be followed during point collection. This document will cover the protocols for collecting GPS and photo information to determine storm water levels using smartphone technology.

Using your smartphone outdoors allows the internal GPS to connect to satellites. Give your phone enough time to connect to satellites before collecting an observation. This could take 10-15 minutes. Your collection can be made without cellular service, however, the accuracy of your position will be reduced.

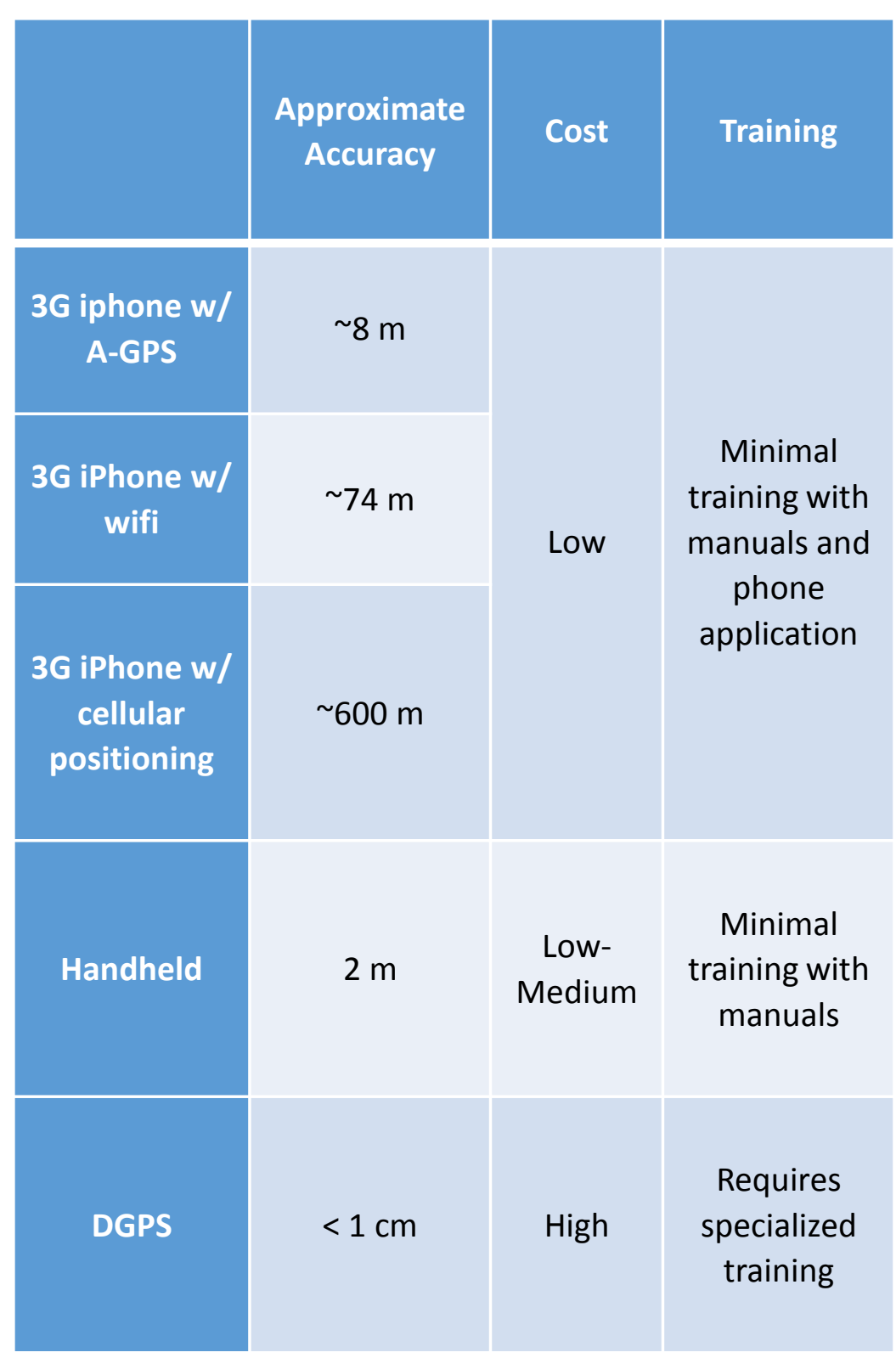

(Wing, 2008; Zandbergen, 2009) 


\section{Data Collection Procedure}

When an observation is posted in the LEO Network, the phone application will prompt the user to enter specific information about an observation. The following steps provide guidance on how to enter this information for observations of flooding.

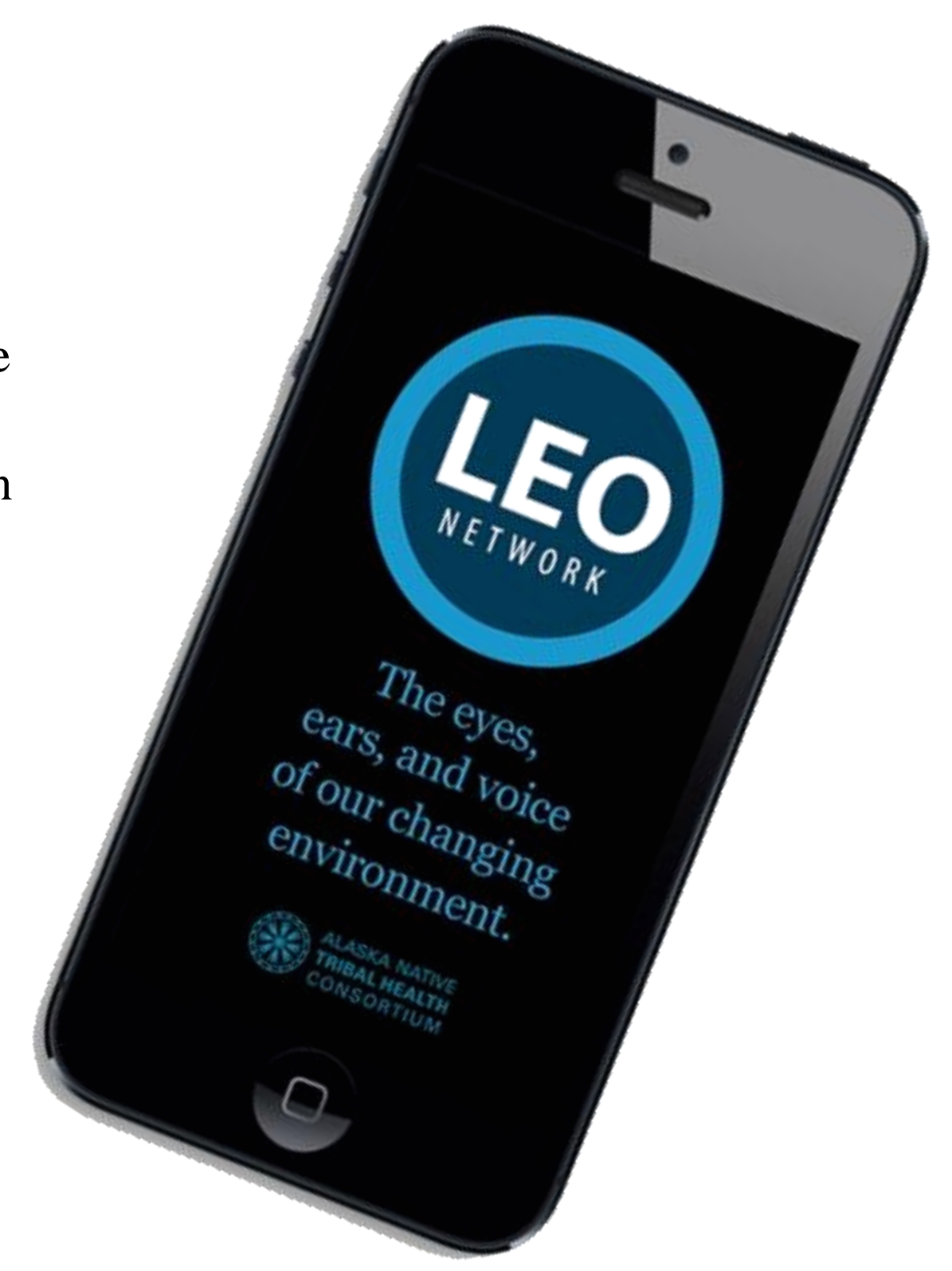


Consider where you are observing the floodwaters and what processes might be taking place. If you live on a spit or barrier island, there may be ocean on either side of your community. These two sides, ocean and lagoon or river system, usually have different types of flooding. Estimates of flood elevations on both sides is useful for future forecasts.

- Ocean-Floodwaters are the result of the combination of storm surge, tides, and waves running up on the beach.

Ocean

- Lagoon or River-Flood waters are the result of storm surge, tides, and water piling up as it exits the mouth of the lagoon or river system.

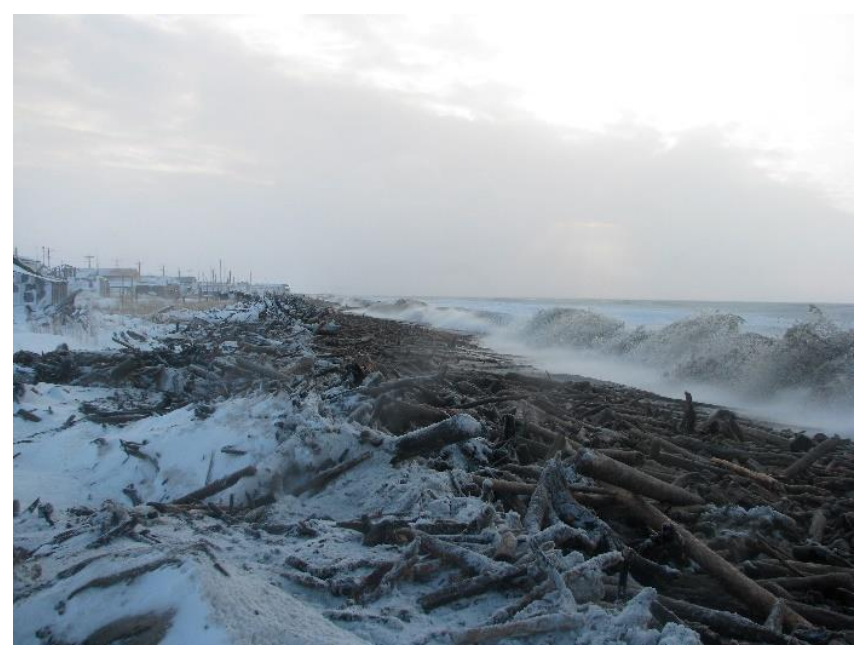

Ocean Side

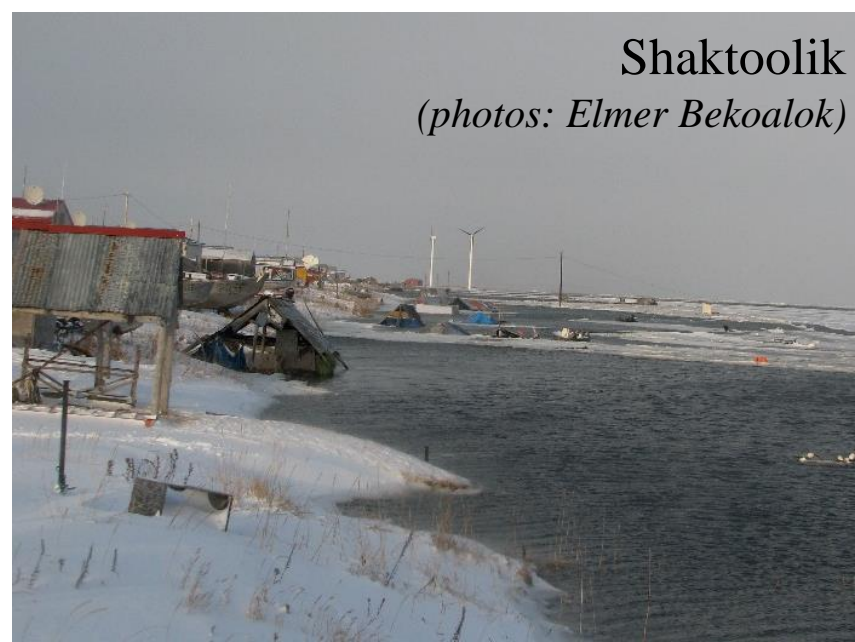

Lagoon Side 


\section{What is your headline?}

\section{Flooding}

\section{When?}

Today >

Where? Current Locati... >

\section{Location \& Time}

There are a few ways of documenting flooding using the phone application. It is best to collect as much information as possible, but if you are limited by time, select the method that works best for you.

- Single Point - Observations provided relative to a known point; location can be surveyed in at a later date.

- Multiple Points - Observations recorded for multiple points provides information about the distribution of flood elevations and reduces uncertainty in maximum flood elevation.

Observations must be taken directly after the storm event to preserve the visible feature being measured. Waves and activity in the nearshore environment often obscure these features very quickly (USACE, 2012). 


\section{Single Point Observation}

If you are only able to collect a single point to associate with storm flooding, it is best to do so relative to a reference point. A reference point is a photo-identifiable feature that can be found later and surveyed. This, however, requires a surveyor to visit the location of flooding at a later date, which is not always possible.

"When photographing high water marks, a close up of the water mark with a tape measure showing the distance from a reference point (windowsill, floor elevation, etc.) will validate a high water elevation long after the mark has disappeared." (USACE, 2012)

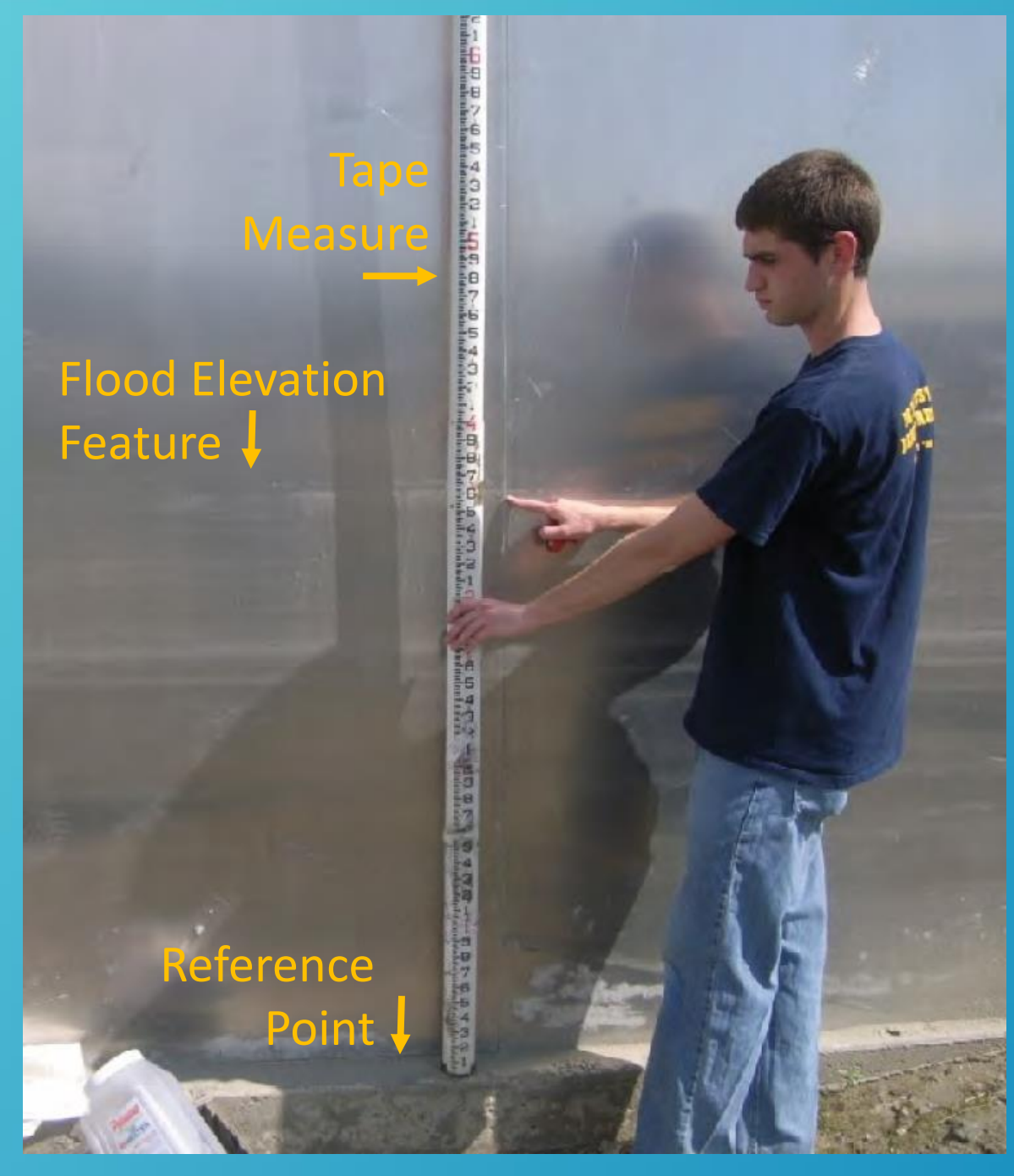

Silt line on aluminum pump house, Russian Mission, Alaska 2009, selected from USACE (2012) 


\section{What is your headline?}

\section{Flooding}

\section{When?}

Today >

\section{Where? Current Locati... >}

\section{Multiple Point Observation}

The information necessary for determining the severity of a flooding event is a high water mark caused by the flooding (USACE, 2012), which, in the coastal environment, can result in:

- Slush Line - Storm wave or surge transported ice slush to the maximum flood elevation.

- Snow/Ice Melt Line - Storm wave or surge melted existing snow or ice to the maximum flood elevation.

- Debris/Wrack Line - Storm wave or surge transported debris or vegetation to the maximum flood elevation.

- Water Line - Storm surge water transported to the maximum flood elevation while the flood is still occurring (If safe to do so).

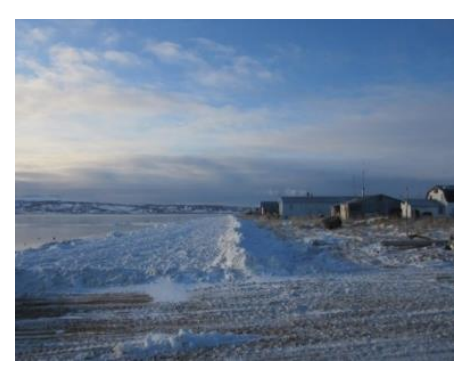

Slush Line

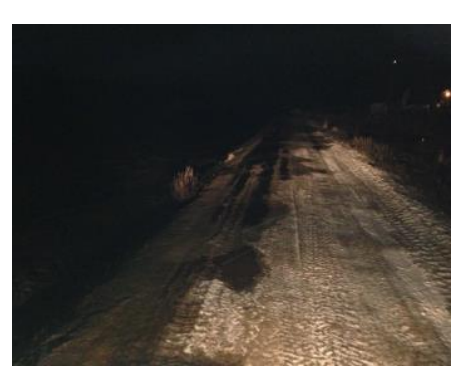

Snow/lce Melt Line

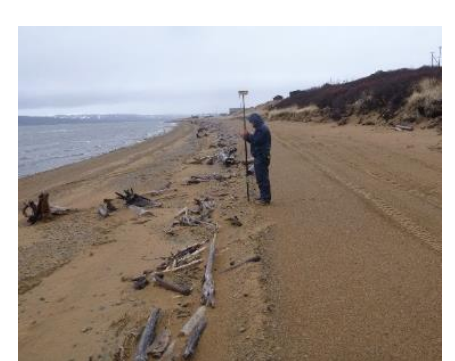

Debris/Wrack Line

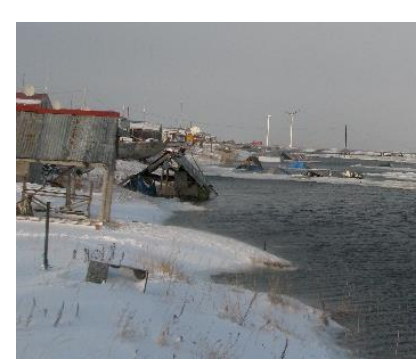

Water Line 


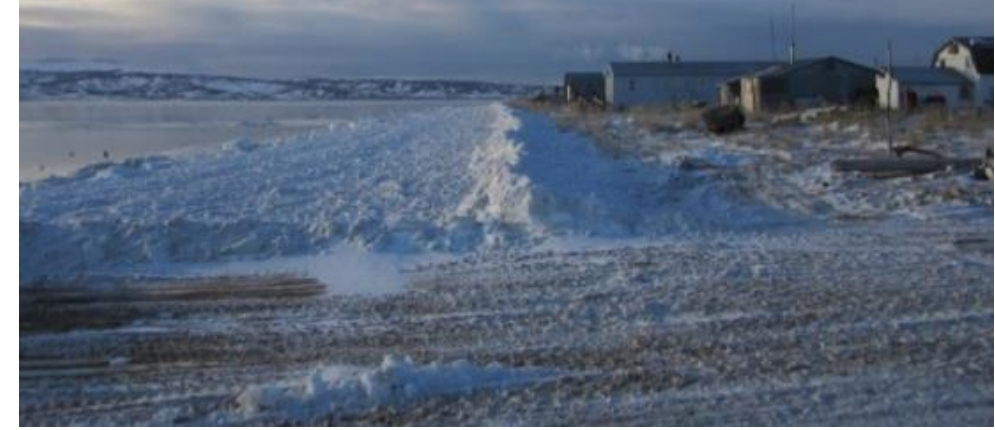

Slush Line

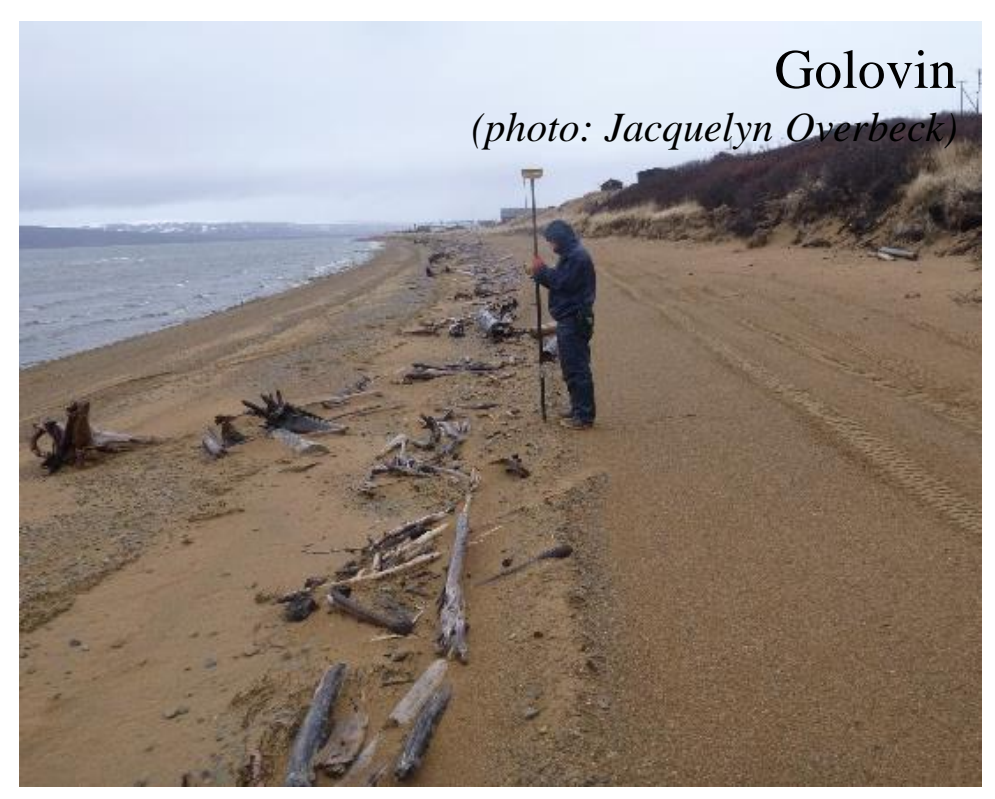

Debris/Wrack Line
Golovin (photo: Harvey Sookiayak)
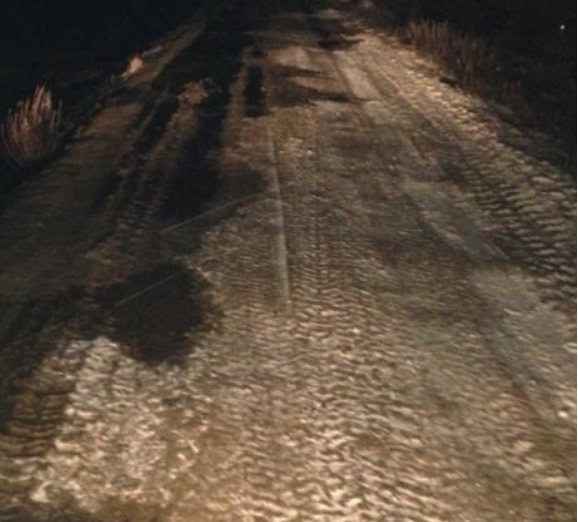

Snow/lce Melt Line

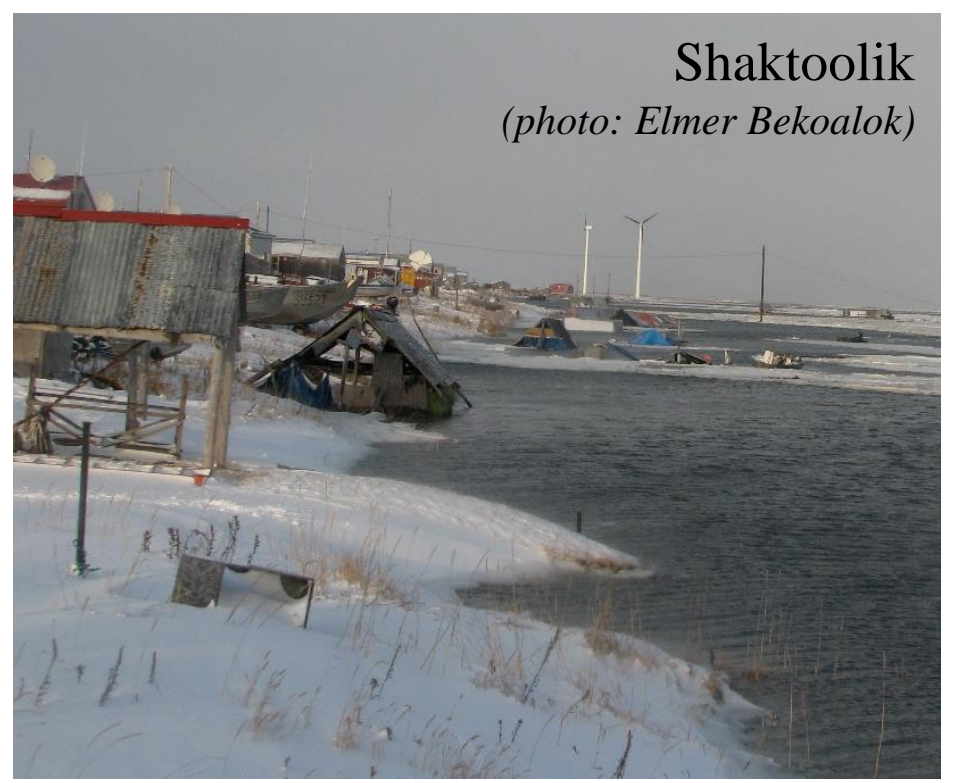

Water Line

\section{Flooding}

What is your headline?

When?

Today

Where? Current Locati... > 


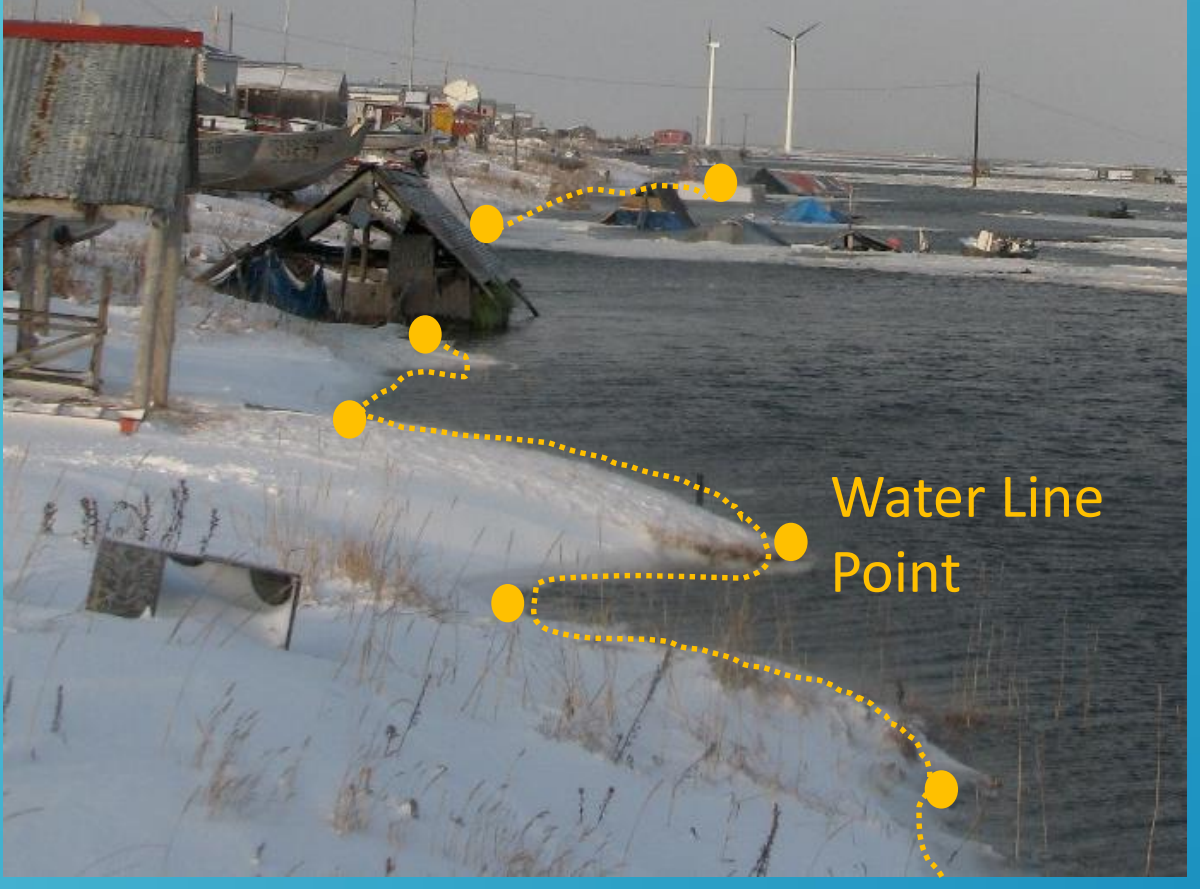

\section{Multiple Point Observation}

If the observed flood feature is a line, multiple GPS points are necessary to determine the maximum flood elevation. These points can be collected in succession, but require multiple observations. Make your initial observation at a single point and fill out fully. If you are collecting the same feature, walk a few meters ( $\sim 10$ feet $)$, then create a new observation without detail in the observation. For the observation text of the consecutive points, write "see original observation". Continue this for the extent of flooding or until you can no longer collect observations. Collect at least three observations in your line.

To ensure your location is accurate, make your observation in an open, flat area, free of buildings and telephone poles. Tall features often block satellite connections and can also obstruct the elevation model being used to interpret the maximum flood elevation. 


\section{Notes About Your Observation}

Other information is helpful for identifying when and why the flood event occurred. And, although you may be familiar with the location of a photo relative to community infrastructure and geographic features, other users will need more explanation. Please include information about:

- Location-Community name, which side of the community ocean/lagoon, the closest building nearby.

- Feature - There may be multiple features in your photo, but explain whether you are collecting a water line, slush line, or something else. If you are collecting a single point, include the tape measure reading.

- Timing - If you are unable to collect information during a storm, please note the timing of the maximum flooding.

- Severity of the Flooding - Low water crossings flooded, minor street flooding, home/business flooded, widespread flooding, etc.

- Other Environmental Factors - Wind direction, whether sea ice was present and in what form, how large the waves were. 
Add images that tell your story

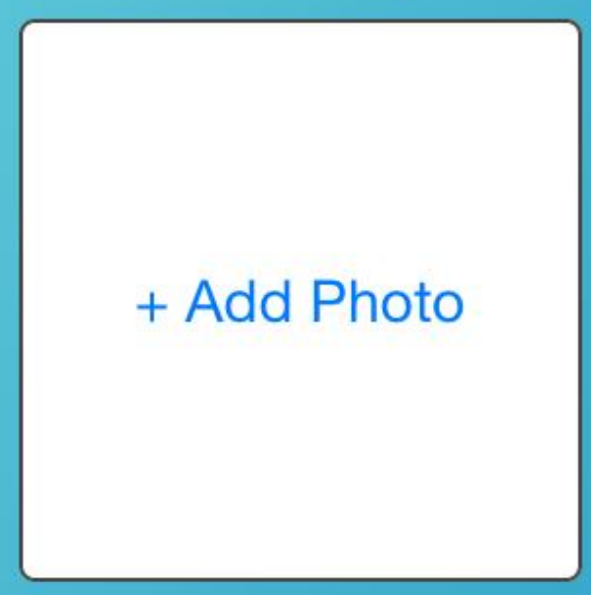

\section{Adding Photos}

Photos are a key component of your observation! Whether you are collecting a single point or multiple points, a photo of your observation confirms exactly what feature you measured, and where it occurred.

Please use the same format as the photos used throughout this document. If you observed a linear feature, take the photo so that you can see down the line with the ocean on one side and land on the other. If you are documenting a single point, be sure to include a tape measure above or below your reference point.

With permission from the observer, these photos may be used in government and/or scientific reports to describe the event. 
Look over your observation and press send!

The LEO Network will route your observation to the project leads who will combine all information from a single event and report back to you with the results.

\section{Ready to send your observation?}

This is a preview of what you are about to send. If you like it, tap the Send button.

\section{Flooding}

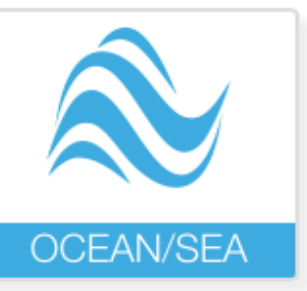




\section{Extracting Flood Elevations from Observations}

The Alaska Division of Geological \& Geophysical Surveys (DGGS) will be using elevation models to translate the latitude and longitude of GPS locations to ground elevations. Aerial surveys conducted over the west coast of Alaska in 2015 (example below) will allow DGGS to perform these measurements. For the full spatial extent of elevation data available in coastal regions, visit http://maps.dggs.alaska.gov/elevationdata/.

Observation Points

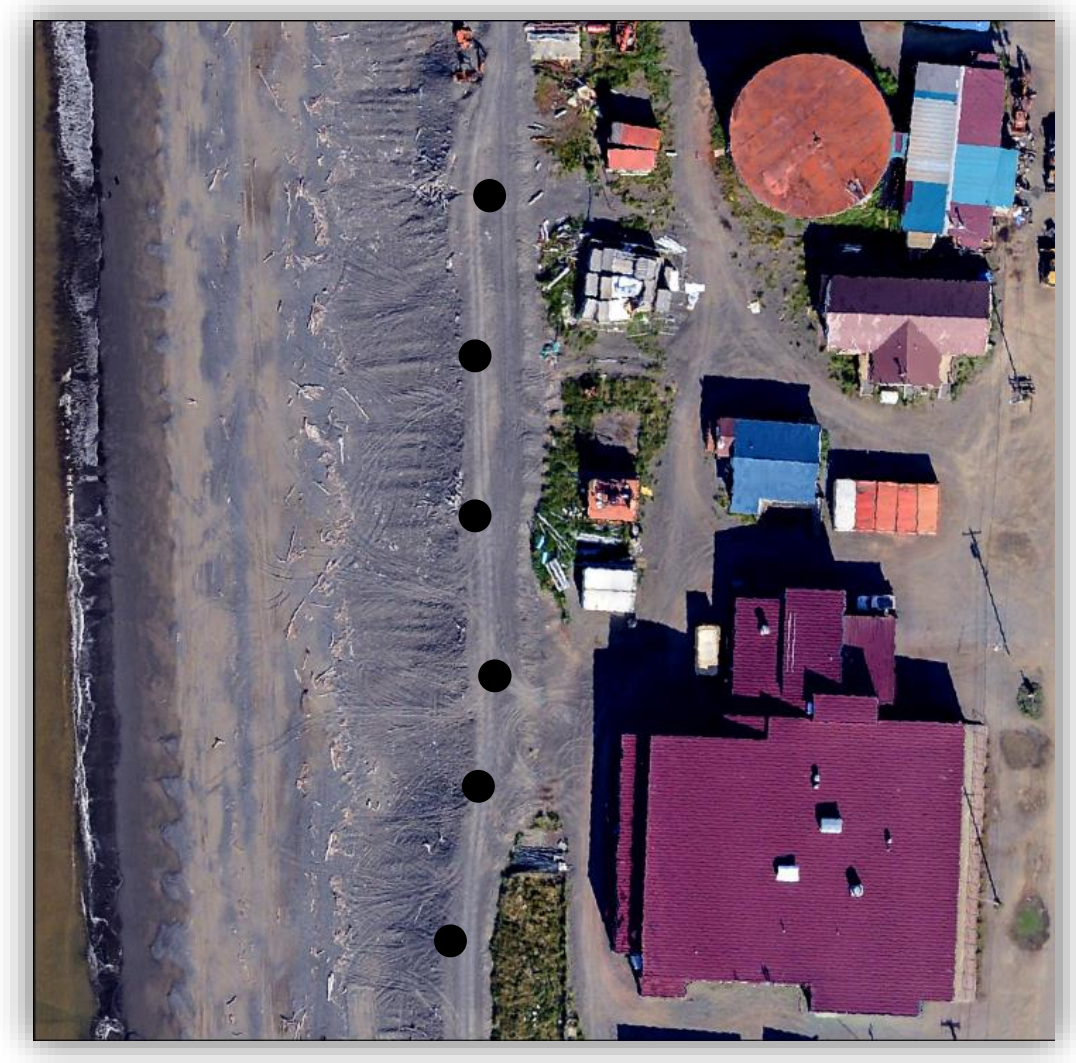

Extracted Elevations

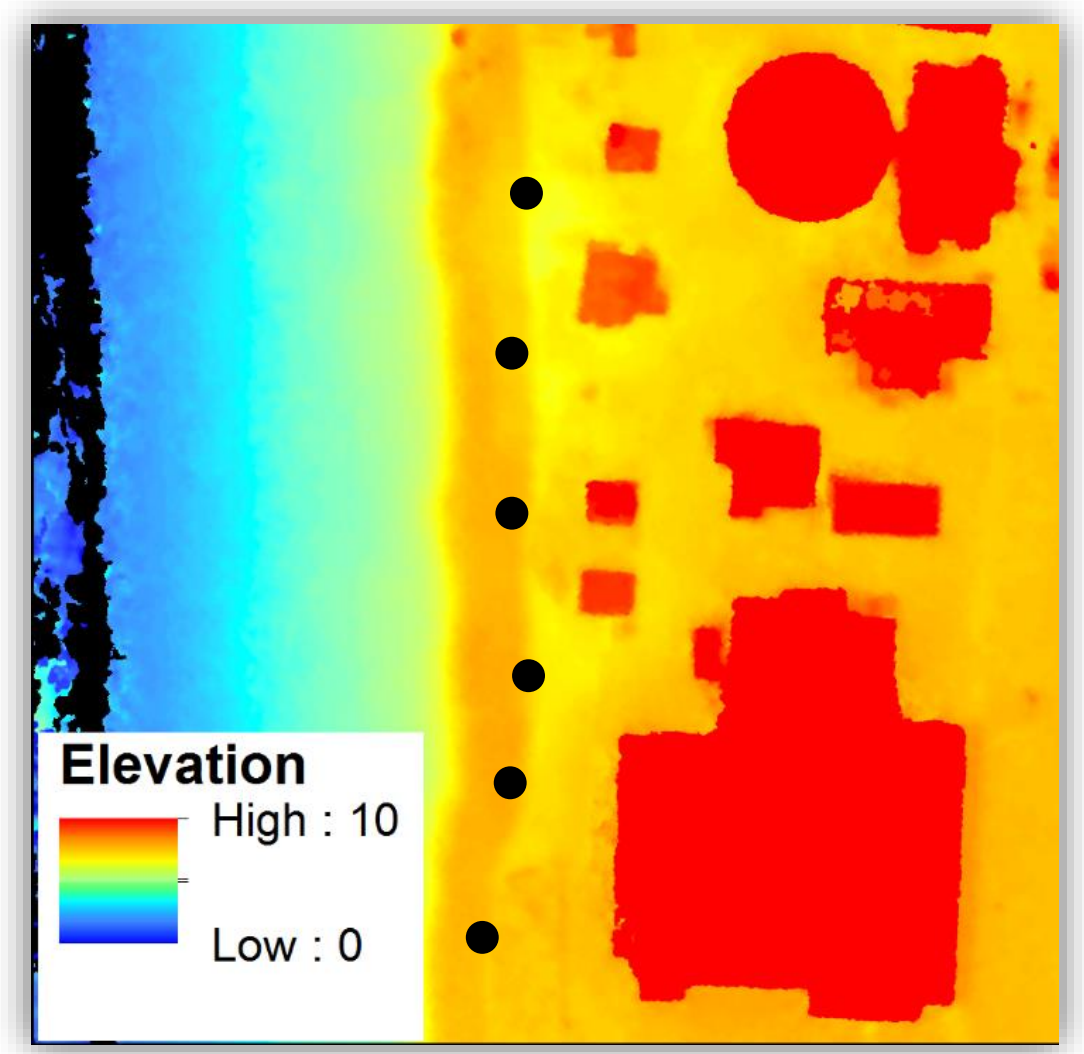

Distribution of Elevation

Observations

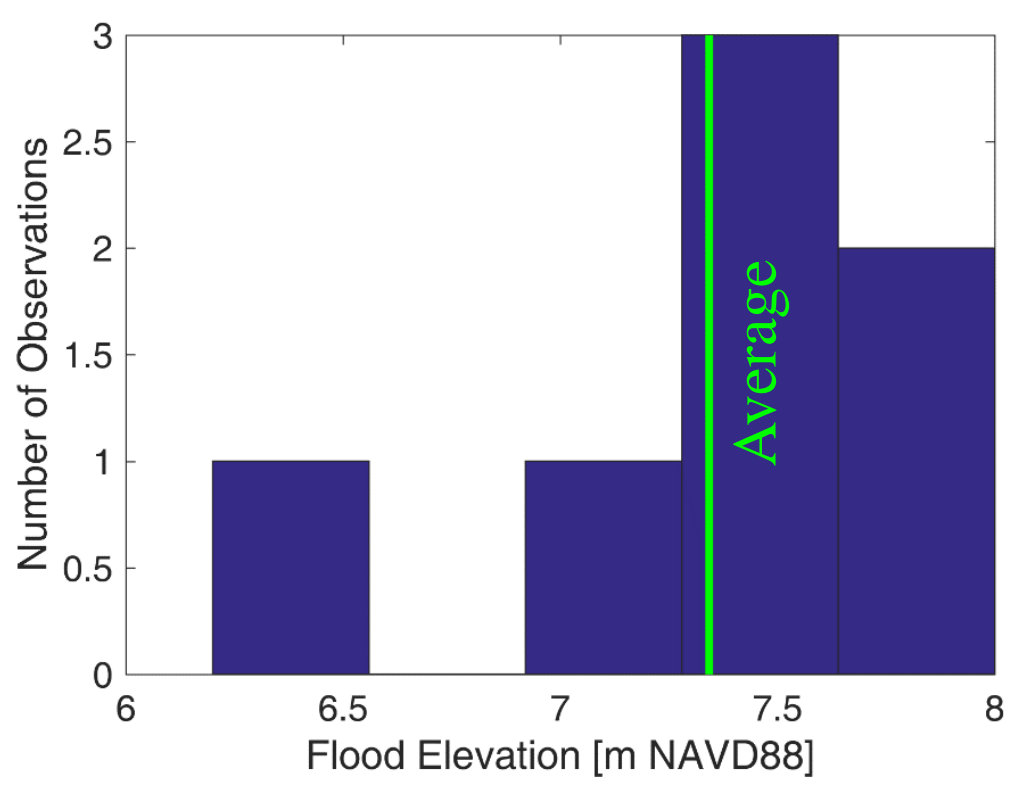

*This example is not from a real flood event. 


\section{Requests for Data}

At any time, you can request the final results of the data you collected from the Alaska Division of Geological \& Geophysical Surveys Coastal Hazards Program. Please allow time for processing and interpreting the data.
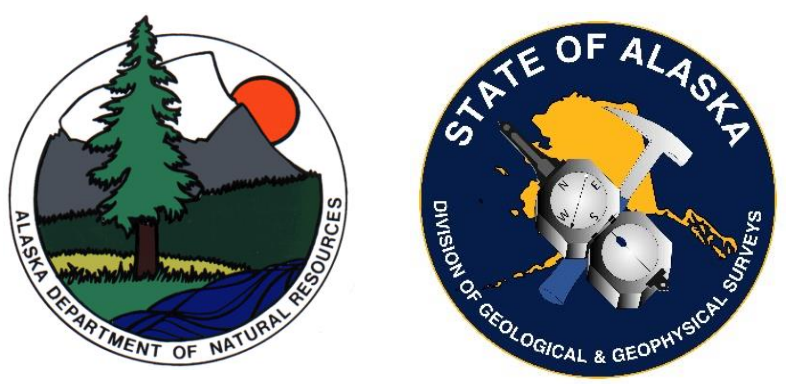

State of Alaska

Department of Natural Resources

Division of Geological \& Geophysical Surveys

\section{Coastal Hazards Program}

Jacquelyn Overbeck

jacquelyn.overbeck@alaska.gov

907-451-5026

http://dggs.alaska.gov/ \\ GEOLOGIC MATERIALS CENTER}

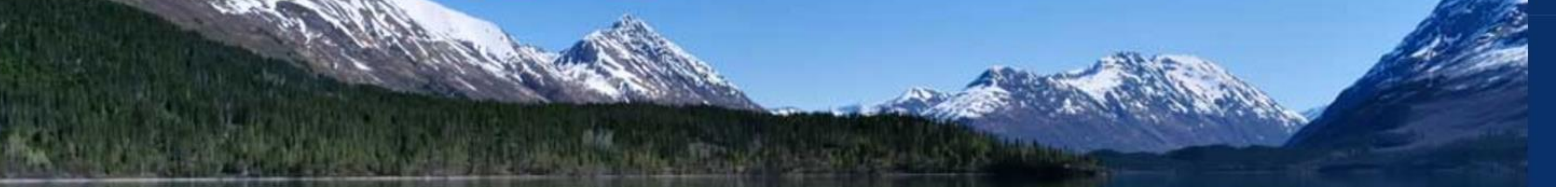

State of Alaska > Department of Natural Resources > Divison of Geological \& Geophysical Surveys > Engineering Geology

COASTAL HAZARDS

Alaska's extensive shorelines an in

Prograts

respond to hazardous events and long-term changes. This program is dedicated to fostering scientific partnerships that will improve the

quality and quantity of the critical baseline data that are necessary to fuel informed decision-making throughout the state.

We routinely respond to information requests by agencies and the general public. If we can assist you in any way please contact our program directly

\section{HEADLINES}

- Online data distribution, educational outreach, and innovative observation strategies for severe

a focus in 2015-2016

- Coming soon:

- New release of aerial photos and elevation models over west coast communities

Island

- The Alaska Tidal Datum Portal has been updated with [new content for 2016]

STAFF Jacquelyn Overbeck Program Manager Alex Gould Geologist RESEARCH INTERNS
Lauren Graduate Intern
Southerland Richard Rummler Undergraduate December 2015 WALCC webinar on the Alaska Shoreline Change Too

TOPICS OF INVESTIGATION

Geohazard Evaluation and Geologic Mapping for Coastal Communities + Overview

+ Focus Communities

+ Publications and Data Products

+ Partners and External Resources

Coastal Topography

+ Overview

+ Featured Projects 


\section{Acknowledgments}

This publication is funded with qualified outer continental shelf oil and gas revenues by the Coastal Impact Assistance Program, U.S. Fish and Wildlife Service, U.S. Department of the Interior. The views and conclusions contained in this document are those of the authors and should not be interpreted as representing the opinions or policies of the U.S. Government. Mention of trade names or commercial products does not constitute their endorsement by the U.S. Government.

Funding for the continuation of this work is provided by Alaska Ocean Observing Systems. Contributions were also made by reviewers whom supplied valuable comments and feedback including Nicole Kinsman and Davin Holen.

AOOS Alaska Ocean Observing System

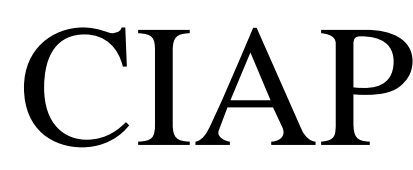

Coastal Impact Assistance Program

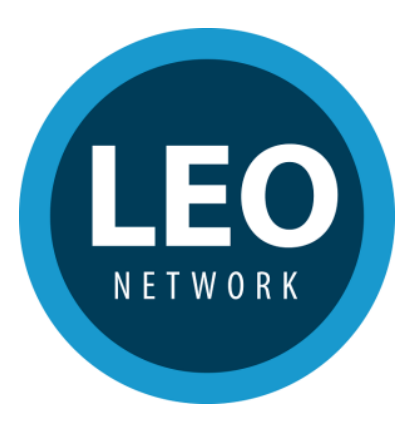

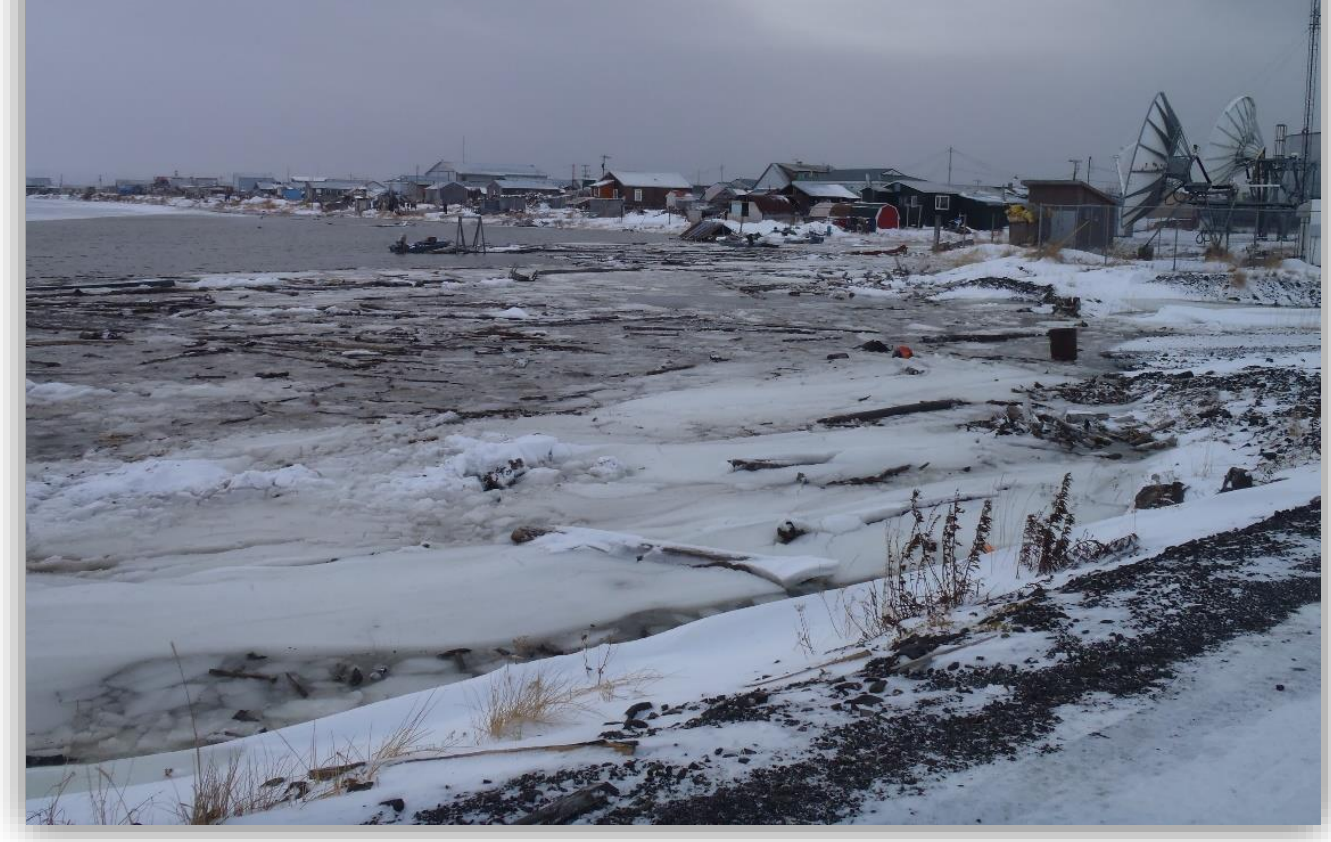

\section{References}

Local Environmental Observer Network (LEO), 2016, The Local Environmental Observer (LEO) Network The eyes, ears, and voice of our changing environment, from: https://www.leonetwork.org/en/leo/about

U.S. Army Corps of Engineers, 2012, Flood and Erosion Data Gathering Procedures: A Guide to Gathering Essential Data After Flooding and Erosion Events: U.S. Army Corps of Engineers, Alaska District.

Wing, Michael G., 2008, Consumer-Grade Global Positioning System (GPS) Receiver Performance: Journal of Forestry, vol. 106, no. 4, pp. 185-190(6).

Zandbergen, Paul A., 2009, Accuracy of iPhone Locations: A Comparison of Assisted GPS, WiFi and Cellular Positioning: Transactions in GIS, Vol. 13 Issue s1, pages $5-25$. 